\title{
AXIAL ABERRATIONS OF LENSES
}

\author{
By E. D. Tillyer and H. I. Shultz
}

\section{CONTENTS}

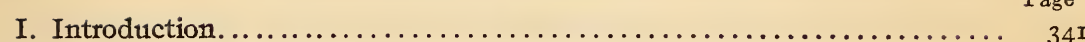

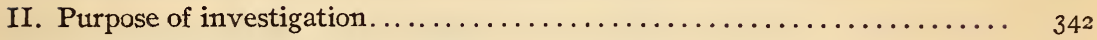

III. Errors affecting an optical image............................ 342

I. Axial chromatic aberration ............................ 343

2. Oblique chromatic aberration...................... 343

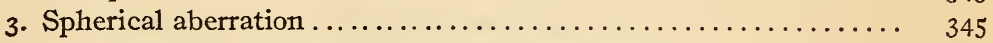

Variation of spherical aberration with color........... 346

4. Zonal variation of equivalent focal length (sine condition)...... 346

Variation of sine condition with color................ $35 \mathrm{I}$

5. Curvature of field.................................. $35^{\mathrm{I}}$

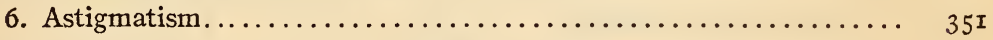

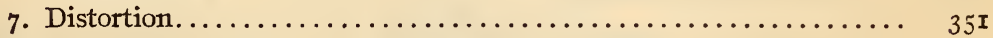

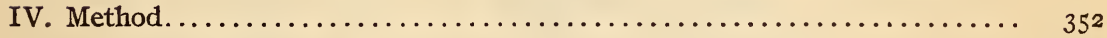

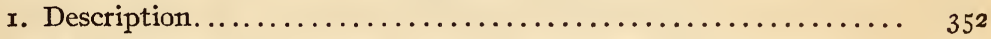

2. Method of manipulation............................. 354

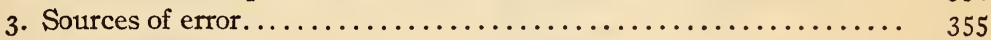

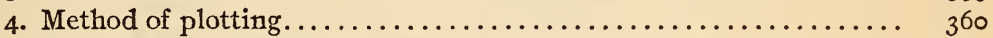

5. Applicability of method.......................... $36 \mathrm{I}$

6. Illustrative description of a set of curves............... 362

V. Classification of curves.................................. ${ }_{36} 6_{3}$

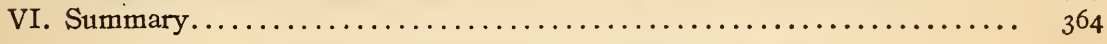

\section{INTRODUCTION}

The definition in the center of the field of a lens system is generally assumed to be good, but its relative importance in different lens systems varies greatly. In a telescope objective, where only the center of the field is used, the errors there require strict correction, while those which affect only the edge of the field can be neglected. In a projection lens the same is true, but to a lesser degree, since more of the field is used than in a telescope objective. Here the central corrections are important, though often neglected because of the low cost of the lens and because the observer sitting at some distance from the projected picture does not see small imperfections in the image.

In photographic lenses a large field is very desirable-really a requirement-and more attention must be paid to the definition at the edge of the field than in the previously mentioned cases. 
Here a compromise must often be made between the center and the edge of the field. In these systems the color must be well corrected to avoid chemical focus or the blurring of the image, due to the actinic rays focusing nearer to (or farther from) the lens than the visual. Especially is the color correction important in lenses used for color photography and in process lenses.

In astrographic lenses, those used in stellar photography, a field is required which is large relative to telescopic systems but small compared with lenses for ordinary photography. Over this field they are required to be corrected very accurately with regard to all aberrations, though color is of secondary importance, since a color screen, or plates which are sensitive only to a short range of the spectrum, may be used.

In portrait lenses the central definition is of greater importance than that of any other part of the field, since focusing on the eyes of the sitter will give sharp definition there and produce the effect of concentrating the attention on the expression without directing it to less important details.

In all cases the central definition is very important and the determination of the errors in the center of the field will generally give a comparative value of the lens. The central corrections show, with very few exceptions, the highest quality of definition a lens will give. Even in a lens with a large field, when a compromise must be made between the center and edge of the field, the central definition must be at least as good as the edge, if not better.

\section{PURPOSE OF INVESTIGATION}

The following investigation was undertaken to determine the errors of a complex lens system which affect the definition of the image near the center of the field, and to compare the various types of lenses on the market with respect to their corrections for central definition and their applicability to various purposes for which they might be used. The lenses investigated were in the following principal classes: Photographic lenses of relatively large aperture; projection lenses for stereopticon and motion picture work; telescope lenses of short focal length and large aperture and telescopic systems.

\section{ERRORS AFFECTING AN OPTICAL IMAGE.}

It seems advisable to define and describe the errors found in optical systems before proceeding to discuss the method used in determining them. Each one of these errors is called an aberra- 
tion, there being seven principal aberrations, namely: (I) Axial chromatic, (2) oblique chromatic, (3) spherical aberration, (4) zonal variation of equivalent focal length (sine condition), (5) curvature of field, (6) astigmatism, and (7) distortion.

I. Axial Chromatic Aberration.-When a ray of white light (Fig. I) passes through the lens $L L$ at $Q$ a prism action is exerted on the ray and the white light is broken up into its component elements. The red component, or the ray of longer wave length, is brought to a focus at a point $P_{1}$, farther from the rear lens surface than the violet component or the ray of shorter wave length, which is focused at $P_{2}$. The intervening wave lengths are focused between the points $P_{1}$ and $P_{2}$. This error is known as axial

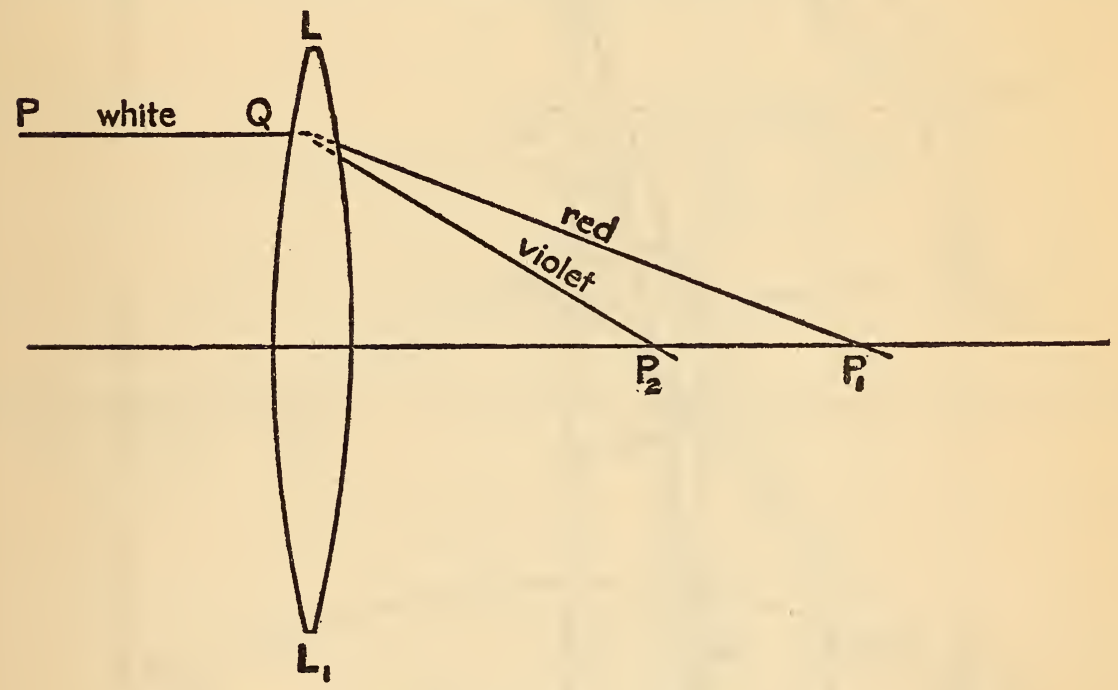

FIG. I.-Diagram illustrating axial chromatic aberration

chromatic aberration. The amount of chromatic aberration, or the distance $P_{1} P_{2}$, is dependent upon the focal length of the lens and the dispersion of the glass. By combining two lenses of proper focal lengths and glasses of suitable refractive indices and dispersions the axial chromatic aberration can be minimizedthat is, two colors such as red and blue-may be brought to a focus at the same point and the other colors be made to focus very close to this point.

2. Oblique Chromatic Aberration.-Even when all the colors are focused at the same point the equivalent focal lengths of the various colors may not be the same, and each color may give a different sized image, causing the familiar colored fringes to large 


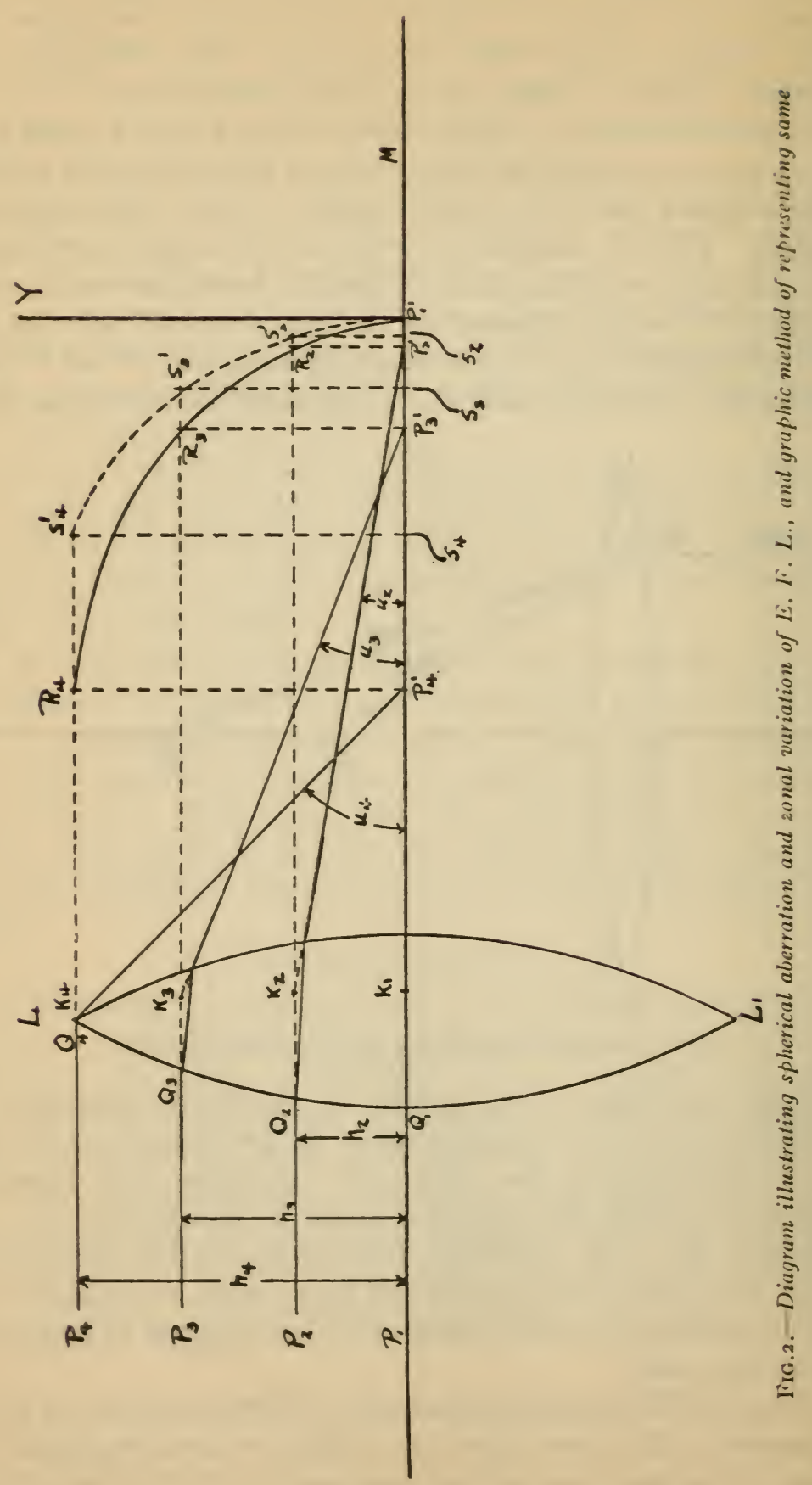


objects. A particular example of this is found in the lenses used in three-color photoengraving, where if the focal length for the red is slightly longer than that for the blue, there will be a red fringe to the image. This error is known as oblique chromatic aberration.

3. Spherical Aberration.-Let $L L_{1}$ (Fig. 2) be a convex lens, with the axis $P_{1} M$, and $P_{1}, P_{2}, P_{3}$, and $P_{4}$ be four parrallel rays striking the lens at points $Q_{1}, Q_{2}, Q_{3}$, and $Q_{4}$ at heights $h_{1}, h_{2} h_{3}$, and $h_{4}$ from the axis. The rays, after being refracted by the lens, will cut the axis at some point. Let the ray from $P_{1}$ traveling infinitely near the axis cut the latter after refraction at $P_{1}{ }^{1}$; then $P_{1}{ }^{1}$ will be the focus for a paraxial ray (ray very near the axis). The ray $P_{2}$, striking the lens at a height $h_{2}$ from the axis will,

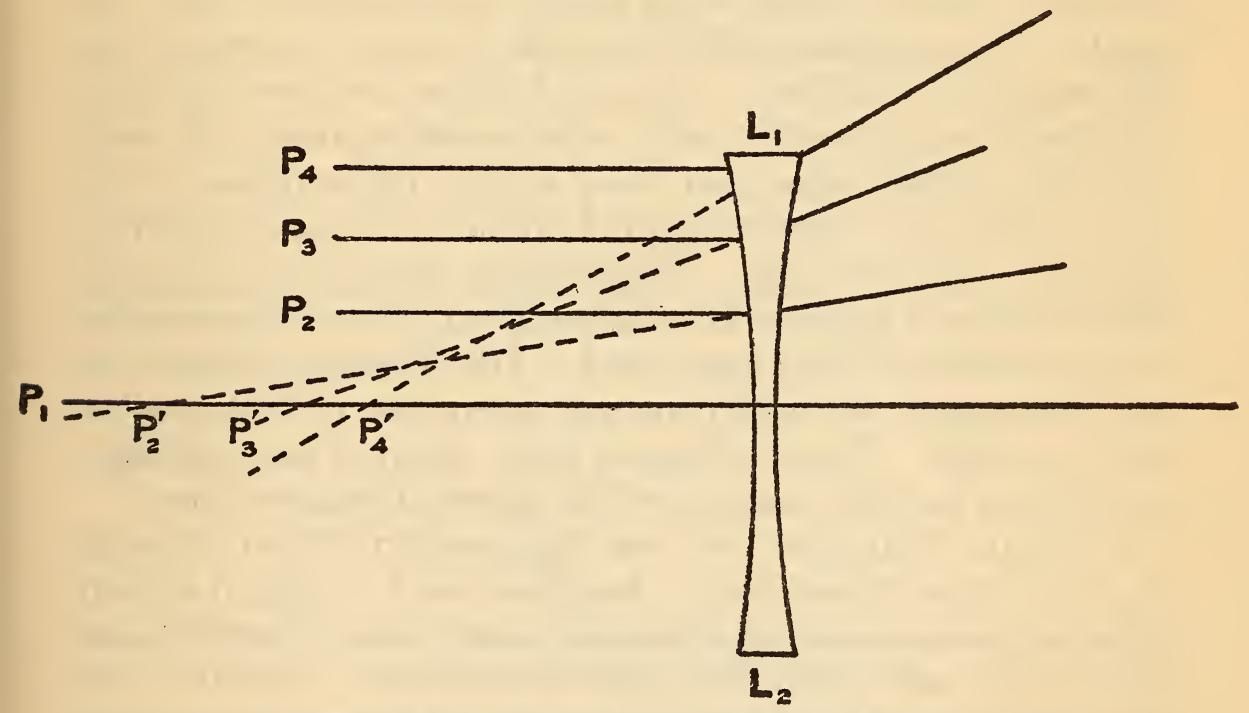

FIG. 3.-Diagram illustrating spherical aberration in a negative lens

after refraction, be brought to a focus at $P^{1}{ }_{2}$. The ray from $P_{4}$, striking the lens at the extreme edge at a height $h_{4}$ from the axis, will be brought to a focus at $P_{4}{ }^{1}$, nearest to the lens. If $V$ represent the paraxial focal length, then $P_{1}{ }^{1} P_{4}{ }^{1}=\Delta V$ is known as the axial spherical aberration.

If we draw $Q_{2} R_{2}, Q_{3} R_{3}$, and $Q_{4} R_{4}$ parallel to the axis, and erect the perpendiculars at $P_{2}{ }^{1}, P_{3}{ }^{1}$, and $P_{4}{ }^{1}$, and draw a curve through the intersections $P_{1}{ }^{1}, R_{2}, R_{3}$, and $R_{4}$, we obtain a curve which represents the spherical aberration along the axis for any zone of the lens at a distance $h$ from the axis.

In a negative lens (Fig. 3 ) the ray through the edge zone, if prolonged backward, would cut the axis at a point $P_{4}{ }^{1}$ nearer the lens than the point $P_{1}{ }^{1}$, which is the focus for paraxial rays. 
But here the spherical aberration $P_{1}{ }^{1} P_{4}{ }^{1}=\Delta V$ is opposite in sign from that of the positive lens. The size of $\triangle V$ is dependent on the shape of the lens, for a given focal length and kind of glass. By combining a positive and a negative lens of the proper shapes and of glasses and focal lengths previously determined by other conditions (axial chromatic, Petzval, and oblique chromatic in photographic lenses, but only axial chromatic in telescope objectives), the spherical aberration of the negative lens may be made to counteract that of the positive lens, and thus be eliminated. In the absence of spherical aberration the curve $P_{1}{ }^{1} R_{4}$ will coincide with the line $P_{1}{ }^{1} Y$.

Variation of Spherical Aberration with Color.-The spherical aberration curves for all colors do not coincide nor do they run parallel to each other, since the axial chromatic aberration for different zones is different. Hence, if curves are drawn for light of different wave lengths, each curve would represent the axial spherical aberration for that wave length, the variation in the shape of the curves representing the variation of the axial spherical aberration with the color. The abscissa of each curve for any definite ordinate is the spherical aberration of the zone represented by that ordinate for the given color. The difference between two such abscissæ is the axial chromatic aberration of that zone for the two colors. Thus, the curves show spherical and chromatic aberrations, and the variation of the spherical with the color.

4. Zonal Variation of the Equivalent Focal Length (E. F. L.) (Sine Condition).-Assume a lens L L (Fig. 4) entirely corrected for spherical and chromatic aberrations. Then all zones will have the same axial focal point for all colors. If all the zones have not the same focal length, then the aberration called coma is present.

Let $P Q$ (Fig. 4) be a small object perpendicular to the axis. Then $P Q$ will be imaged in $P^{\prime} Q^{\prime}$ by the central zone of the lens $L L_{1}$. If the lens is free from spherical and chromatic aberrations, the point $P$ will be focussed by the edge zone as well as by all other zones at $P^{\prime}$; but if the E. F. L. (equivalent focal length) of the different zones be different, the edge zone will have a different magnification from the central zone and the object $P Q$ will be imaged in $P^{\prime} Q^{\prime}$ by the central zone, and in $P^{\prime} Q^{\prime \prime}$ by the edge zone. In other words, the edge zone will focus the point $Q$ in $Q^{\prime \prime}$ in the same plane but in a different position from $Q^{\prime}$, the image by the central zone. If $Q$ be a finite point, then $Q^{\prime}$ will be a very small disk of somewhat larger diameter than the disk pro- 
duced at $Q^{\prime \prime}$. Hence the point $Q$ will be imaged in a figure shaped like a comet, having a bright point at one end and trailing off in a broad fuzzy circle at the other. The sharp point will be directed toward or away from the axis depending on the relative sizes of the focal lengths for the edge and central zones.

The graphical representation of zonal variation of $\mathrm{E}$. F. L. is shown in Fig. 2. Prolong the rays to $P_{1}{ }^{\prime}, P_{2}{ }^{\prime}, P_{3}{ }^{\prime}$, and $P_{4}{ }^{\prime}$ backward into the lens till they meet the rays $P_{1}, Q_{1}, P_{2} Q_{2}, P_{3} Q_{3}$, and $P_{4} Q_{4}$ prolonged at $K_{1}, K_{2}, K_{3}$, and $K_{4}\left(K_{1}\right.$ is obtained by computing the axial focal length and measuring back from $\left.P_{1}{ }^{\prime}\right)$. The points $K_{1}, K_{2}, K_{3}$, and $K_{4}$ are the principal points for the corre-

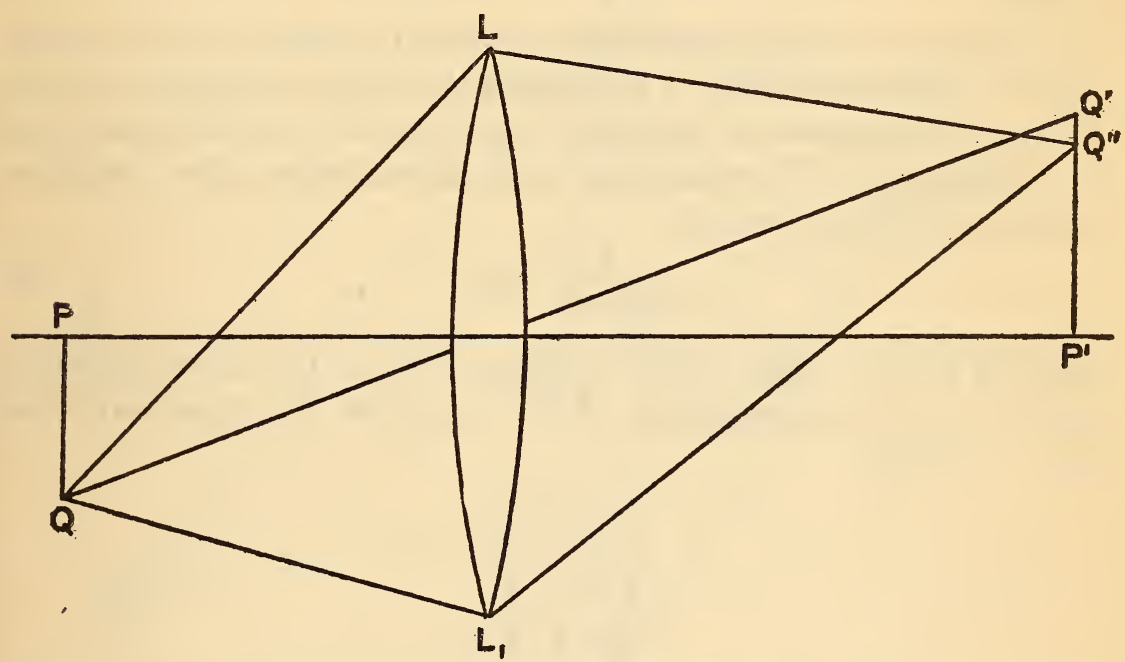

FIG. 4.-Diagram illustrating image by lens free from spherical aberration, but having zonal variation of $E . F . L$.

sponding zones, and the surface on which all these points lie is the principal surface of the lens.

Let $h$ be the height at which the incident ray strikes the lens and $u$ be the angle which the refracted ray makes with the axis. Then $\frac{h}{\sin u}=E$ is defined as the E. F. L. of the zone at a distance $h$ from the axis. In Fig. 2,

$$
\begin{aligned}
& \frac{h_{1}}{\sin u_{1}}=K_{1} P_{1}^{\prime} \\
& \frac{h_{2}}{\sin u_{2}}=K_{2} P_{2}^{\prime} \\
& \frac{h_{3}}{\sin u_{3}}=K_{3} P_{3}^{\prime} \\
& \frac{h_{4}}{\sin u_{4}}=K_{4} P_{4}^{\prime}
\end{aligned}
$$


will be the equivalent focal lengths of the corresponding zones. If the lens is absolutely free from spherical aberration, then the condition

$$
\frac{h}{\sin u}=\text { constant }
$$

means also freedom from zonal variation of the E. F. L.; hence freedom from coma for points near the axis. Equation $(a)$ is commonly known as the "Sine condition" for rays from an infinitely distant object. For artificially stopped systems and at large angles with the axis other additional terms have to be considered. ${ }^{1}$

In practice, no lens is entirely corrected for spherical aberration. Some of the latter is left in to balance the higher order aberrations and to compromise for the best condition for the elimination of the other errors. In this case, as is shown below, the condition for freedom from coma is

$$
\frac{h}{\sin u}-f=0
$$

where $f$ for the zone $h$ is the distance from the axial principal point $K_{1}$ to the intersection of the ray from that zone with the axis. In Fig. 2

$$
\begin{aligned}
& f_{1}=K_{1} P_{1}^{\prime} \\
& f_{2}=K_{1} P_{2}^{\prime} \\
& f_{3}=K_{1} P_{3}^{\prime} \\
& f_{4}=K_{1} P_{4}^{\prime}
\end{aligned}
$$

Let $L L$ (Fig. 5) be a lens with spherical aberration present. Then the rays from the edge and center will intersect the axis at $P^{\prime}$ and $P^{\prime \prime}$, respectively. Assuming $\frac{h}{\sin u}=$ constant, every zone will have the same magnifying power, and there will be a series of images of the object $P Q$ at all points from $P^{\prime}$ to $P^{\prime \prime}$, all of the same size. But the position of the plate will be the position of the average image somewhere between $P^{\prime}$ and $P^{\prime \prime}$, say, $G G$. The image at $P^{\prime \prime} Q^{\prime \prime}$ for the edge zone, when produced forward to the plate $G G$, will become a large disk in the position $\mathrm{mm}^{\prime}$. The image for the central zone produced back to $G G$ will be a small disk at $m^{\prime \prime}$. At $Q^{n}$ there will be a sharp image produced by some zone between the center and the edge of the lens. Following the

\footnotetext{
1 The greatest effect on the definition is due to these seven errors; but there are certain unnamed aberrations, consisting of higher order terms, which affect the image to a smaller extent. Czapski gives $x 2$ terms of the fifth order and 20 terms of the seventh order, besides the five terms of the third order.
} 
same procedure, the other zones will produce disks of diameter smaller than $\mathrm{mm}^{\prime}$. The figure on $G G$ will consist of a bright point at $m^{\prime \prime}$ trailing off into the broad fuzzy perimeter of the disk $m m^{\prime}$, giving the true shape of coma.

In order to obtain a symmetrical image in a lens with spherical aberration present, the series of out-of-focus images in any position $G G$ (Fig. 6), formed by producing the rays of the various images

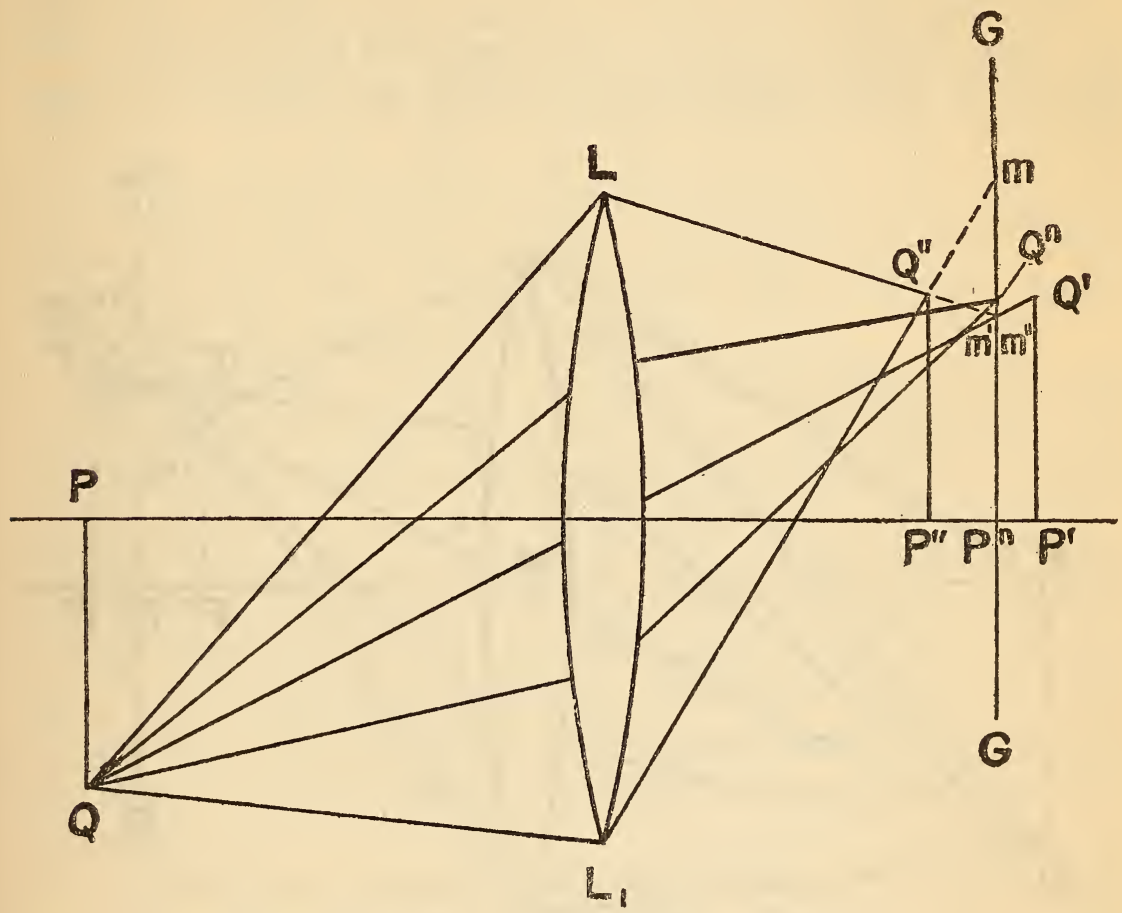

FIG. 5.-Diagram illustrating image by lens possessing spherical aberration, but free from zonal variation of E. F. L. (Condition $\frac{h}{\sin u}=$ const. fulfilled)

$Q^{\prime}$ to $Q^{\prime \prime}$, must all be symmetrically placed with respect to the ray $K Q^{\prime}$, through the central zone. In Fig. 6,

$$
\frac{K P^{\mathrm{n}}}{K P^{\prime}}=\frac{P^{\mathrm{n}} Q^{\mathrm{n}}}{P^{\prime} Q^{\prime}}
$$

but

and

$$
\frac{P^{\mathrm{n}} Q^{\mathrm{n}}}{P^{\prime} Q^{\prime}}=\frac{\frac{h}{\sin u_{\mathrm{n}}}}{\frac{h_{\mathrm{1}}}{\sin u_{1}}}=\frac{E_{\mathrm{n}}}{E^{\prime}}=\frac{E_{\mathrm{n}}}{E_{\mathrm{ax}}}
$$

$$
K P^{\prime}=F_{\text {ax }}, K P^{\mathrm{n}}=f_{\mathrm{n}}
$$

where the subscript "ax" refers to the axial zone. 
Hence,

For the axial zone,

$$
\frac{f_{\mathrm{n}}}{f_{\mathrm{ax}}}=\frac{E_{\mathrm{n}}}{E_{\mathrm{ax}}}
$$

$$
E_{\mathrm{ax}}=f_{\mathrm{ax}}=K_{1} P_{1}^{\prime} \text {. }
$$

Hence $E_{\mathrm{n}}-f_{\mathrm{n}}=\mathrm{O}$ is the condition for a symmetrical image free from comatic flare in a lens with spherical aberration present.

If we plot along the axis $P^{\prime} Y$ (Fig. 2) the height $h$, and along $P^{\prime} P$ the variation $\Delta E$ in the equivalent focal lengths of the zones from that of the central zone, we obtain the curve $P^{\prime} S^{\prime}$ repre-

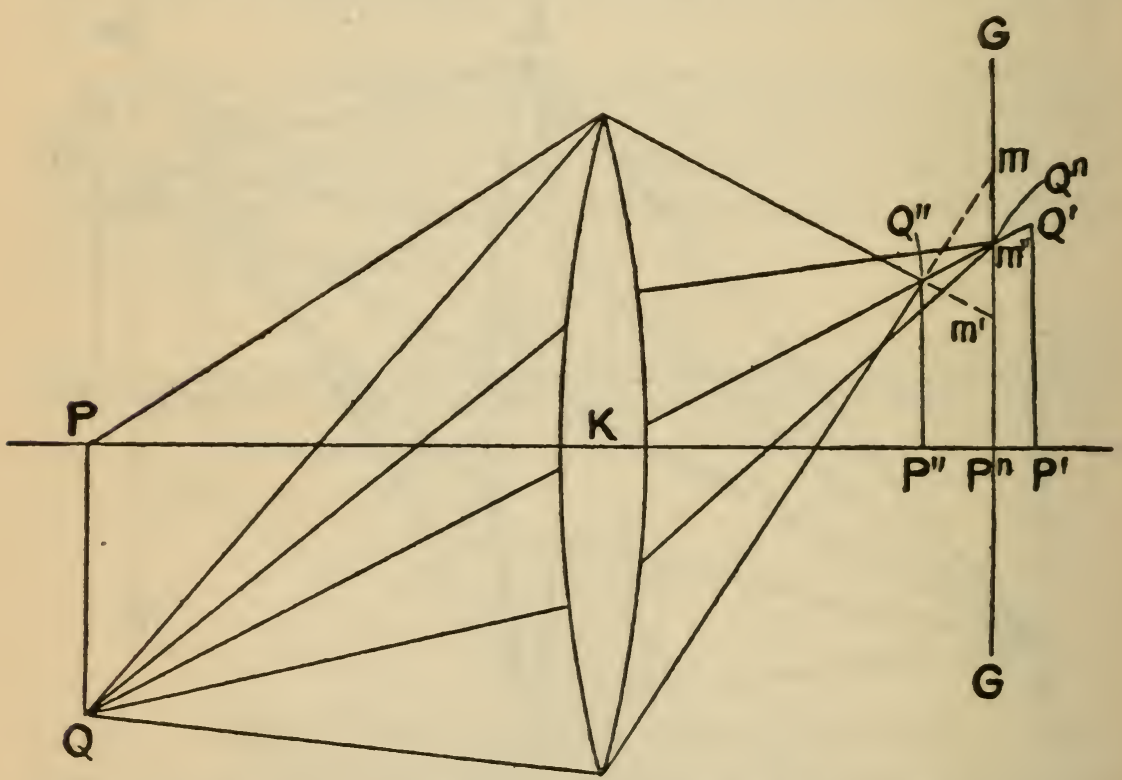

FIG. 6. - Diagram illustrating image by lens with condition $\left(\frac{h}{\sin u}-f=0\right)$ fulfilled

senting the zonal variation of the $\mathrm{E} . \mathrm{F}$. L. If we represent the E. F. L. of the zone $h$ by $E_{\mathrm{b}}$ and that of the axial zone by $E_{\mathrm{ax}}$, then

$$
\Delta E=E_{\mathrm{b}}-E_{\mathrm{ax}}
$$

Similarly, if $f_{a x}$ is the distance from the paraxial principal point to the paraxial focal point and $f_{\mathrm{h}}$ is the distance from the same principal point to the focal point for the zone $h$, then the spherical aberration $\Delta V=f_{\mathrm{h}}-f_{\mathrm{ax}}$.

When expressed in terms of the aberrations $\Delta E$ and $\Delta V$, the condition $E_{\mathrm{h}}-f_{\mathrm{h}}$ becomes

$$
E_{\mathrm{h}}-f_{\mathrm{h}}=\left(E_{\mathrm{ax}}+\Delta E\right)-\left(f_{\mathrm{ax}}+\Delta V\right)=\Delta E-\Delta V,
$$

since $E_{\mathrm{ax}}=f_{\mathrm{ax}}$. 
In Fig. 2 the full line represents $\Delta V$ and the dotted one is $\Delta E$, hence the condition for freedom from coma for points near the axis, $\Delta E-\Delta V=O$, means that the two curves coincide.

Variation of Sine Condition with Color.-If the zonal variation of the E. F. L. be determined for different wave lengths, the curves may not coincide, showing a variation of $\Delta E$ with the color. Since the size of the image depends on the E. F. L., the zonal variation of the E. F. L. with color gives the variation of the size of the image with the color and hence the oblique chromatic aberration.

5. Curvature OF FIELD.-The preceding aberrations are the ones to be given most consideration when examining the central definition of a lens. When we depart somewhat from the center of the field and go toward the edge, we find that the image of a plane object does not lie in a plane. If we plot the points of best focus, we find them forming a surface of a saucerlike shape. This defect is known as curvature of field.

6. Astigmatism.- - Toward the edge of the field we find that there may not be a position of good focus. The image may consist of a series of streaks running radially, and somewhere in a plane other than that of the radial image another series of streaks in a direction perpendicular to the first. This is due to the fact that a pencil of rays from a point striking the lens in one plane focuses at a point different from the focus of a pencil of rays from the same point but in a plane perpendicular to that of the first pencil. This error is known as astigmatism, and is nonexistant in the center of the field of a lens with good surfacing and not strained in the mounting.

7. DisToRTION.-Another aberration which affects the image toward the edge of the field is distortion. This is the deformation of a straight line in the object into a curved line in the image due to a variation of the angular magnifying power of a lens for rays incident at different angles.

Besides the preceding aberrations, we must take into account the so-called hybrid aberrations, or the effect of some of the previously mentioned errors on the others. These affect the definition at the edge of the field and are exceedingly important in photographic lens systems where a large field is very desirable.

For the purpose of ascertaining the best use to which a lens of a given description may be put it is necessary to determine the magnitude of these aberrations, and the region of best correction. The significance of the axial aberrations from the point of view of 
general definition has been discussed in a previous paragraph. Hence the determination of these axial aberrations will give us a comparative value of the lens. To do this the method described below was devised.

\section{METHOD}

The method used is an extension of Hartmann's method of objective testing. ${ }^{2}$ His work is fully described in the Zeitschrift fur Instrumentenkunde. Hartmann uses a screen to isolate rays along four diameters, $45^{\circ}$ apart. He takes shadowgraphs at points very near the focus and from these he obtains the spherical aberration and the astigmatism along the axis. For the zonal variation of the $\mathrm{E} . \mathrm{F}$. L. he places the lens with the mount in contact with a scale and measures the apparent length of the scale divisions through the lens with a micrometer. Hartmann's method has been applied to many large telescope objectives and small lenses. The method is tedious and complicated and requires two totally independent sets of measurements to obtain both the spherical aberration and the zonal variation of the E. F. L. Moreover, for comparatively thick lenses it is not possible to use the method for the zonal variation of the E. F. L. The extension described later gives both the errors with a single measurement and can be used for all kinds of lenses of relatively large aperture and short focal length and for complete telescopic systems.

I. DesCRIPTION.-Fig. 7 is a diagrammatic sketch of the method. Parallel rays from a distant source fall on a metal screen $S S$ pierced with small holes which isolate single rays. These rays are then refracted by the lens system under test, $L L$, and come to a focus at $F$, or near $F$, in the presence of spherical aberration. A photographic plate is placed in the position $A$ and a shadow photograph taken. The plate is then moved to the position $B$ on the other side of the focus and another shadow photograph taken. The shadow photographs have center difraction disks which can readily be measured if care is taken to choose a screen with holes of proper diameter and distance apart. If the plate is near the focus, as at $B$, sharp disks are obtained; at $A$, farther away from the focus, the disks are less sharp.

Consider a ray, $R$ (Fig. 7), passing through the screen and striking the lens at a height, $h$, from the center. It is refracted by the lens system making a disk on the plate $A$ at $m$, and on the plate $B$ at $n$. If $M$ and $N$ are the positions of the disks made by

2 Objectiountersuchungen., Zeitshrift fur Instrumentenkunde, 24, pp. 1, 33, 97; r904. 
the ray through the center of the lens, then $a$ and $b$ will be the distances from the center of the plates $A$ and $B$ to the intersections of the ray $R$ with the plates. The position $F$ will be given by the expression $V=\frac{a d}{a+b}$ where $V$ is the distance $M F$ from the fixed point $M$, and $d$ is the distance between the two positions of the plates. Assuming that $V_{\mathrm{o}}$ is the distance of the paraxial focal

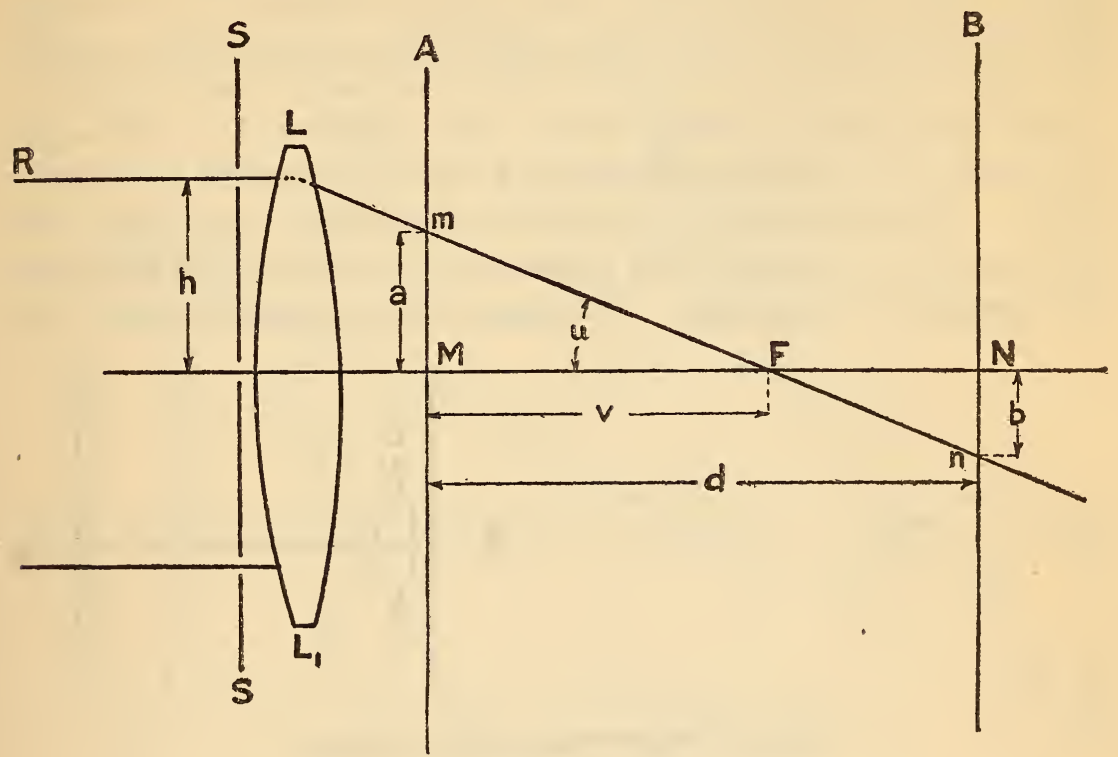

FIG. 7.-Diagrammatic sketch of methods

point from $M$, then the spherical aberration for the zone at a distance, $h$, from the lens axis is $\Delta V_{\mathrm{h}}=V_{\mathrm{h}}-V_{\mathrm{o}}$.

In Fig. $7 u$ is the inclination of the refracted ray with the axis and

$$
\tan u=\frac{a+b}{d}
$$

from which $\sin u$ is easily determined. Hence, $\frac{h}{\sin u}$ for each zone can at once be determined if $h$ is known. For this purpose, the screen used was calibrated and the position of the holes was known to within $0.005 \mathrm{~mm}$.

The apparatus used consisted of the following: $R$ (Fig. 8) is a monochromatic source of light, which in this case was a Fuess monochromator giving a fairly pure band of the spectrum of about $4 \mu \mu$ in width in the violet $(425 \mu \mu)$ to about $21 \mu \mu$ in the red $(650 \mu \mu)$. 
The illuminant was a Nernst filament close to the slit. The width of the band of spectrum was determined with a Hilger wave-length spectrometer, which had been checked up by means of a mercury arc. The monochromator was placed about 20 feet away from the screen, and corrections applied to reduce the results to infinity. The screen $S$ was a metal plate accurately centered with respect to the lens holder. The holes used for fairly large-sized lenses were $\mathrm{I} \mathrm{mm}$ in diameter and spaced at intervals of $3 \mathrm{~mm}$ apart. For smaller lenses a screen with smaller holes spaced closer together was used, permitting a suitable number of zones to be determined. If the holes are too small, or too near together, the diffraction disks will not be clearly defined and it will be impossible to measure them. The lens holder, $L$, is of the iris diaphragm type, so that the lens is always centered with respect to the holder, and therefore with respect to the screen. The lens holder rotates about a ver-
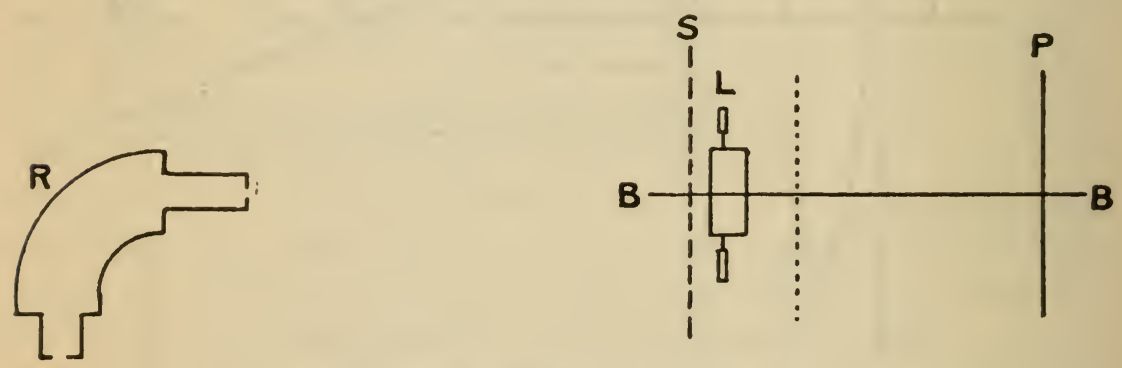

FIG. 8.-Diagrammatic sketch of apparatus

tical axis, permitting the lens to be centered with respect to the photographic plate. The photographic plate $P$ slides on an optical bench $B B$ along a scale. The plate can be raised or lowered, permitting several exposures to be made on the same plate.

2. Method of Manipulation.-The lens is placed in the holder, care being taken that it is fairly well centered. It is then accurately centered by reflection from the back of the lens. For this purpose a ground-glass plate with a little $90^{\circ}$ prism cemented to it (leaving a clear space in the ground glass surrounding the prism) is placed in the plate holder. The prism is used to send a beam of light to the rear lens surface. When the beam is reflected on itself by the lens and comes back to the observer through the clear space around the prism, then the lens is normal to the direction of motion of the plate, and the centering is accurate.

The monochromator is then set to give a beam of wave length $425 \mu \mu$, the plate placed at a considerable distance in front of the 
focus and an exposure made. The plate is then lowered, the monochromator changed to give a beam of wave length $475 \mu \mu$ and another exposure made. This is repeated with the monochromator set at $550 \mu \mu$ and $650 \mu \mu$. The plate is then moved through a distance $d$, previously determined, so that the plate now comes behind the focus. The exposures are repeated through the wave lengths $650 \mu \mu, 550 \mu \mu, 475 \mu \mu$, and $425 \mu \mu$. The length of exposure is timed so as to get uniform density on the plate for all colors, giving disks which can easily be measured on a Zeiss horizontal com-

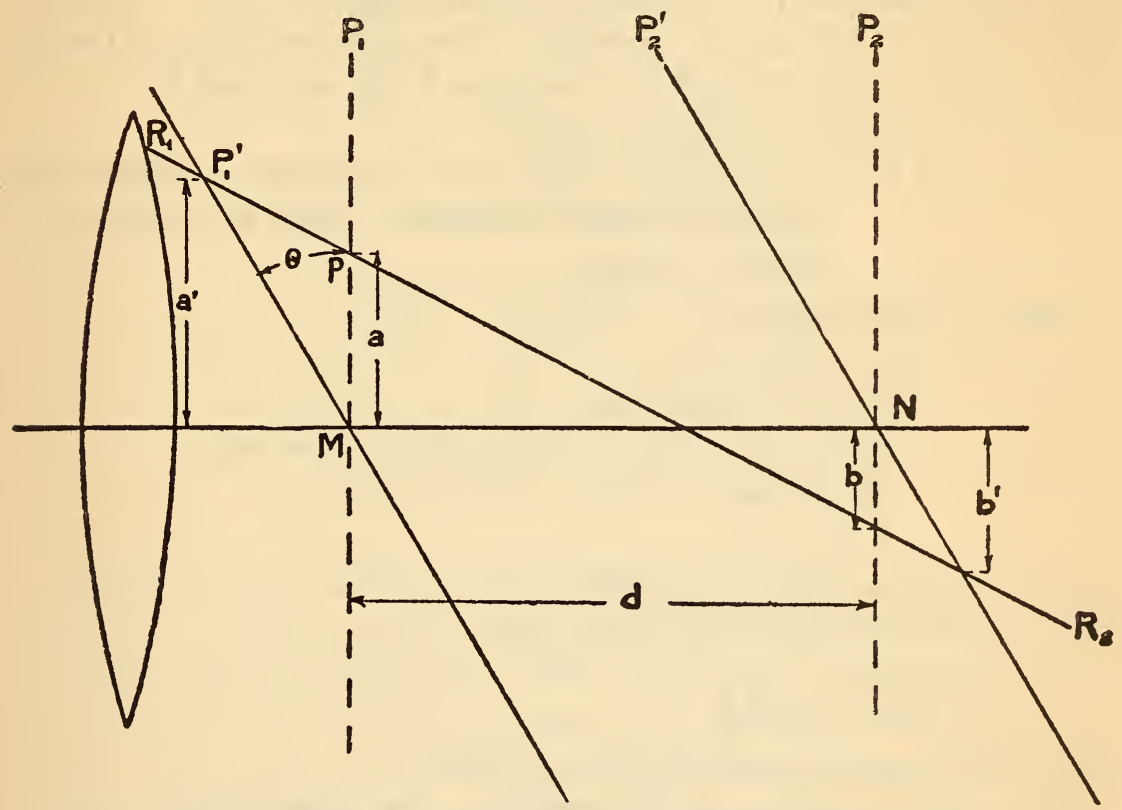

Fig. 9.-Diagram illustrating a tilt of the plate to the lens axis

parator or measuring engine with sufficient accuracy for rays somewhat removed from the axis of the lens.

3. SOURCES OF ERROR.-The lens was carefully centered in the method described above. A slight tilting of the plate would produce an error in $\Delta V$ and $\Delta E$ of at least the second order.

To find the effect of a slight tilting of the photographic plate on the value of $V$, let us assume that $\theta$ be the angle that the plate $P^{\prime}{ }_{1}$ (Fig. 9) makes with the perpendicular to the lens axis $P_{1} M$, which position the plate should occupy. The distance $M F=V$ $=\frac{a d}{a+b}=\frac{a^{\prime} d}{a^{\prime}+b^{\prime}}$, which shows that there is no error produced in $V$ 
by a tilt in the plate through an angle $\theta$ to the perpendicular to the axis, and hence no errors in $\Delta V$.

The effect of the tilt $\theta$ of the plate on the value of $E$ can be determined as follows:

$E=\frac{h}{\sin } u$, but for purposes of discussing the errors $\tan u=\frac{a+b}{d}$ can be substituted for $\sin u$ so that $E=\frac{h}{\tan u}=\frac{h d}{a+b}$

Let the plate be in the position $P_{1}^{\prime} M$ (Fig. 9) making an angle $\theta$ with the position $P_{1} M$ where the plate ought to be. The distance $d=M N$ will not be affected. The only quantities entering in $E$ which will be affected by the angle $\theta$ will be $a$ and $b$.

Let $P_{1}^{\prime} M=a^{\prime}$, as measured, and

$p M=a$, the distance from $M$ to the point $p$ where the ray $R_{1} R_{2}$ would strike the plate in its perpendicular position.

Then, in the triangle, $P_{1}^{\prime} P_{1} M$

$$
\begin{aligned}
& \angle P_{1}^{\prime} M p=\theta \\
& \angle M P P_{1}^{\prime}=(90+u) \\
& P P_{1}^{\prime} M=180-(90+u)-\theta=90-(u+\theta) \\
& M P_{1}^{\prime}=a \\
& P M=a^{\prime}
\end{aligned}
$$

then $\frac{a}{a^{\prime}}=\frac{\sin (90+u)}{\sin [90-(u+\theta)]}=\frac{\cos u}{\cos (u+\theta)}$

$$
a=a^{\prime}\left(\frac{\cos u}{\cos (u+\theta)}\right)
$$

A similar expression can be derived for $b$.

The angle $u$ in any case is never large, the largest angle obtainable in an $f: 2$ lens is one whose tan is one-fourth, which is about $13^{\circ}$. The angle $\theta$ is very small, due to care taken in centering. Hence, $\cos u$ is not very different from $\cos (u+\theta)$ and $a$ may be taken equal to $a^{\prime}$. Similarly, $b=b^{\prime}$. Hence, the error in $E$ due to a tilt of the photographic plate may be neglected.

The error in $\Delta E$ is still smaller, as $\Delta E$ is the difference between two quantities in which the error is systematically in the same direction.

The error in $\Delta E$, due to an error in the known value of $h$, is one of the first order, as $E=\frac{h \text {. }}{\sin u}$. The metal screen used was calibrated so that the value of $h$ was known to within $0.005 \mathrm{~mm}$, corresponding to an average error in $E$ of less than 0.05 per cent. 
An error in the distance $d$ affects both $V$ and $E$ systematically in the same direction.

$$
\begin{aligned}
& V=\frac{a \quad d}{a+b} \\
& E=\frac{h \quad d}{a+b}
\end{aligned}
$$

Assuming an error in $d$, equal to $\delta$, then the spherical aberration, $\Delta V$ and the zonal aberration $\Delta E$ of any two zones will be, respectively,

$$
\begin{aligned}
& \Delta V=\frac{a_{1}(d+\delta)}{a_{1}+b_{1}}-\frac{a_{2}(d+\delta)}{a_{2}+b_{2}} \\
& \Delta V=\left(\frac{a_{1}}{a_{1}+b_{1}}-\frac{a_{2}}{a_{2}+b_{2}}\right) d+\left(\frac{a_{1}}{a_{1}+b_{1}}-\frac{a_{2}}{a_{2}+b_{2}}\right) \delta \\
& =\text { spherical }+ \text { sperical } \cdot \frac{\delta}{d} \\
& \Delta E=\frac{h_{1}(d+\delta)}{a_{1}+b_{1}}-\frac{h_{2}(d+\delta)}{a_{2}+b_{2}} \\
& \Delta E=\left(\frac{h_{1}}{a_{1}+b_{1}}-\frac{h_{2}}{a_{2}+b_{2}}\right) d+\left(\frac{h_{1}}{a_{1}+b_{1}}-\frac{h_{2}}{a_{2}+b_{2}}\right) \delta \\
& =\text { zonal variation }+ \text { zonal variation } \cdot \frac{\delta}{d}
\end{aligned}
$$

Hence the percentage error in the spherical aberration and in the zonal variation of the E. F. L., due to an error in $d$, is the same as the percentage error in $d$. The value of $d$ in all the lenses measured lies between 200 and $400 \mathrm{~mm}$, and the error in $d$ is about o.I $\mathrm{mm}$. This gives a percentage error of from 0.05 to 0.02 per cent, which is negligible. In the following discussion of errors we will neglect the effect of an error in $d$.

For a discussion of the effect of an error in $a$ and $b$ on the final results, we have the formulas

$$
\begin{gathered}
V=\frac{a \cdot d}{a+b} \quad \tan u=\frac{a+b}{d} \quad E=\frac{h}{\sin u} \\
\partial V=\frac{a \partial a+b \partial a-a \partial a-a \partial b}{(a+b)^{2}} d+\frac{a}{a+b} \partial d
\end{gathered}
$$

Neglecting $\partial d$ and substituting tan $u$ for $\frac{a+b}{d}$

$$
\partial V=\frac{b \partial a-a \partial b}{(a+b)} \cdot \frac{\mathrm{I}}{\tan u}
$$


From (I) we see that $\partial V$ is dependent on $\partial a$ and $\partial b$. In practice $\partial a$ and $\partial b$ are smaller, the smaller $a$ and $b$, but negligibly so, so that for an approximation $\partial a$ and $\partial b$ may be considered independent of the size of $a$ and $b$. Hence, we may assume $\partial a=\partial b$, and $a=b$, approximately; then, since $\partial a$ and $\partial b$ may be either plus or minus,

$$
\partial V=\frac{1.4 a \partial a}{2 a} \cdot \frac{\mathrm{I}}{\tan u}=\frac{1.4 \partial a}{2 \tan u}=\frac{1.4 \partial b}{2 \tan u}
$$

Hence $\partial V$ is dependent on $a+b$, only as the latter is contained in $\tan u$.

$$
\begin{gathered}
E=\frac{h}{\sin u}=\frac{h}{\tan u}=\frac{h d}{a+b} \\
-\partial E=h \cdot d\left(\frac{\partial a+\partial b}{(a+b)^{2}}\right)=\frac{\partial a+\partial b}{(a+b)} \cdot \frac{\mathrm{x}}{\tan u} \cdot h
\end{gathered}
$$

If, now, we assume $\partial a=\partial b$ and $a=b$, then

$$
\partial E=\frac{\mathrm{I} .4 \partial a}{2 a} \cdot \frac{h}{\tan u} \text { or } \frac{\mathrm{I} .4 \partial b}{2 b} \cdot \frac{h}{\tan u}
$$

Hence, the larger $(a+b)$ the smaller $\partial E$, the error in $E$.

$$
\begin{gathered}
(E-V)=\frac{h d-a d}{(a+b)}=\frac{d(h-a)}{a+b} \\
\partial \frac{(E-V)}{d}=\frac{-(a+b) \partial a-(h-a)(\partial a+\partial b)}{(a+b)^{2}}
\end{gathered}
$$

After expanding and collecting terms, this becomes

$$
\partial \frac{(E-V)}{d}=\frac{-(h+b) \partial a-(h-a) \partial b}{(a+b)^{2}}
$$

Again assuming $\partial a=\partial b$, and $a=b$

$$
\begin{gathered}
\partial \frac{(E-V)}{d}=\frac{-h \partial a-a \partial a-h \partial a+a \partial a}{(2 a)^{2}}=\frac{1 \cdot 4(-h \partial a-a \partial a)}{4 a^{2}} \\
\frac{\partial(E-V)}{d}=\frac{-1 \cdot 4(h+a) \partial a}{4 a^{2}}
\end{gathered}
$$

$h$ is about twice the size of $a$, hence

$$
\partial(E-V)=4 \cdot 2 \frac{d \cdot(-a \partial a)}{4 a^{2}}=-\frac{d}{a} \partial a, \text { approximately. }
$$


Hence, an error of $\partial a$ in $a$ or $\partial b$ in $b$ will cause an error in $(E-V)$ equal to $d \frac{\partial a}{a}$ or $d \frac{\partial b}{b}$.

The errors due to measurement and to irregularities in the photographic plate were examined by taking two plates of the same lens and having two observers measure the same plate. The following table gives the results thus obtained. $\quad P_{1}$ and $P_{2}$ are the measurements on plates I and $2, P_{1}-P_{2}$ being their difference. In the column $T-S$ is given the variation between two observers. Because of the physical indeterminateness of the values for the first two zones, they may be disregarded, and the probable error of a single observation, then, is $0.06 \mathrm{~mm}$ in $V$ and $0.04 \mathrm{~mm}$ in $E$ for two plates. There is a slight systematic difference between the two plates, which may be due to different intensities in the shadowgraphs or to a speck of dust changing the shape of the shadowgraph.

The probable error of a single observation for two observers as obtained from the columns $T-S$ is $0.02 \mathrm{~mm}$ for $V$ and $0.05 \mathrm{~mm}$ for $E$. The systematic error here is very slight.

Comparison of Measurements

\begin{tabular}{|c|c|c|c|c|c|c|c|c|}
\hline \multicolumn{5}{|c|}{ V (spherical aberration) } & \multicolumn{4}{|c|}{ E (sine condition) } \\
\hline$P_{1}$ (first & plate) & $\begin{array}{c}\mathbf{P}_{2} \text { (second } \\
\text { plate) }\end{array}$ & $\mathbf{P}_{1}-\mathbf{P}_{2}$ & $\begin{array}{c}\mathrm{T}-\mathrm{S} \\
\text { difference } \\
\text { between } 2 \\
\text { observers }\end{array}$ & $\begin{array}{c}\mathbf{P}_{1} \text { (first } \\
\text { plate) }\end{array}$ & $\begin{array}{c}\mathbf{P}_{2} \text { (second } \\
\text { piate) }\end{array}$ & $\mathbf{P}_{1}-\mathbf{P}_{2}$ & $\begin{array}{c}\mathrm{T}-\mathrm{S} \\
\text { difference } \\
\text { between } 2 \\
\text { observers }\end{array}$ \\
\hline $425 \mu \mu$ & $\begin{array}{r}\mathrm{mm} \\
138.91 \\
8.44 \\
8.26 \\
7.94 \\
7.74 \\
7.84 \\
8.62\end{array}$ & $\begin{array}{r}\operatorname{mm} \\
138.80 \\
8.66 \\
8.40 \\
8.09 \\
7.90 \\
8.02 \\
8.78\end{array}$ & $\begin{array}{c}\mathrm{mm} \\
-0.11 \\
-.22 \\
=.14 \\
-.15 \\
-.16 \\
-.18 \\
-.16\end{array}$ & $\begin{array}{l}\mathrm{mm} \\
-0.10 \\
=.00 \\
=.04 \\
=.00 \\
+.01 \\
+.02 \\
+.01\end{array}$ & $\begin{array}{r}\text { mm } \\
212.82 \\
2.92 \\
2.44 \\
2.36 \\
2.06 \\
2.18 \\
3.02\end{array}$ & $\begin{array}{r}\mathrm{mm} \\
212.61 \\
3.03 \\
2.60 \\
2.40 \\
2.16 \\
2.32 \\
3.15\end{array}$ & $\begin{array}{c}\mathrm{mm} \\
+0.21 \\
=.11 \\
-.16 \\
-.04 \\
-.10 \\
=.14 \\
-.13\end{array}$ & $\begin{array}{l}\operatorname{mm} \\
+0.17 \\
+.13 \\
+.03 \\
=.14 \\
=.10 \\
=.02 \\
-.07\end{array}$ \\
\hline $475 \mu \mu$ & $\begin{array}{l}8.74 \\
8.50 \\
8.14 \\
7.83 \\
7.52 \\
7.52 \\
8.06\end{array}$ & $\begin{array}{l}8.88 \\
8.55 \\
8.35 \\
8.00 \\
7.70 \\
7.65 \\
8.23\end{array}$ & $\begin{array}{l}-.14 \\
-.05 \\
\pm .04 \\
\pm .00 \\
\pm .01 \\
\pm .04 \\
\pm .00\end{array}$ & $\begin{array}{l}-.02 \\
=.07 \\
=.03 \\
=.04 \\
\pm .03 \\
=.04 \\
-.02\end{array}$ & $\begin{array}{l}2.36 \\
2.78 \\
2.44 \\
2.24 \\
1.88 \\
1.92 \\
2.41\end{array}$ & $\begin{array}{l}2.96 \\
2.95 \\
2.67 \\
2.24 \\
1.98 \\
1.96 \\
2.58\end{array}$ & $\begin{array}{r}-.63 \\
=.17 \\
-.23 \\
.00 \\
=.10 \\
=.04 \\
-.17\end{array}$ & $\begin{array}{l}-.50 \\
+.18 \\
-.10 \\
=.08 \\
=.08 \\
-.04 \\
-.01\end{array}$ \\
\hline $550 \mu \mu$ & $\begin{array}{l}8.92 \\
8.90 \\
8.33 \\
7.98 \\
7.57 \\
7.44 \\
7.81\end{array}$ & $\begin{array}{l}9.36 \\
8.74 \\
8.56 \\
8.07 \\
7.72 \\
7.58 \\
7.98\end{array}$ & $\begin{array}{l}-.44 \\
+.16 \\
-.23 \\
=.09 \\
=.15 \\
=.14 \\
-.17\end{array}$ & $\begin{array}{l}+.17 \\
+.13 \\
+.03 \\
+.01 \\
\pm .03 \\
+.03 \\
+.02\end{array}$ & $\begin{array}{l}2.74 \\
3.04 \\
2.69 \\
2.30 \\
1.97 \\
1.81 \\
2.33\end{array}$ & $\begin{array}{l}2.66 \\
2.95 \\
2.76 \\
2.44 \\
2.06 \\
1.94 \\
2.38\end{array}$ & $\begin{array}{l}+.08 \\
\pm .09 \\
-.17 \\
=.14 \\
=.09 \\
=.13 \\
-.05\end{array}$ & $\begin{array}{l}+.62 \\
\pm .38 \\
\pm .20 \\
\pm .02 \\
\pm .10 \\
+.09 \\
+.01\end{array}$ \\
\hline $650 \mu \mu$ & $\begin{array}{l}9.12 \\
9.06 \\
8.65 \\
8.19 \\
7.78 \\
7.56 \\
7.80\end{array}$ & $\begin{array}{l}9.38 \\
9.29 \\
8.91 \\
8.40 \\
7.98 \\
7.69 \\
7.98\end{array}$ & $\begin{array}{l}=.26 \\
=.23 \\
=.16 \\
=.21 \\
=.20 \\
=.13 \\
-.18\end{array}$ & $\begin{array}{l} \pm .06 \\
=.16 \\
=.08 \\
=.05 \\
=.03 \\
=.03 \\
-.01\end{array}$ & $\begin{array}{l}3.72 \\
3.15 \\
3.05 \\
2.52 \\
2.13 \\
1.90 \\
2.32\end{array}$ & $\begin{array}{l}3.58 \\
3.79 \\
3.06 \\
2.60 \\
2.29 \\
1.99 \\
2.32\end{array}$ & $\begin{array}{r}+.24 \\
-.64 \\
=.01 \\
=.08 \\
=.16 \\
-.09 \\
.00\end{array}$ & $\begin{array}{l}-.48 \\
=.09 \\
=.05 \\
\pm .02 \\
=.06 \\
=.04 \\
-.02\end{array}$ \\
\hline
\end{tabular}


The systematic error does not affect the results in any way, as the curve plotted is the difference of all the values from a mean value. In plotting, as described below, the probable error is re-

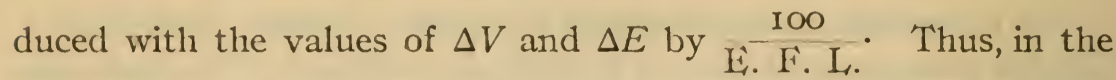
case of a lens of $200 \mathrm{~mm} \mathrm{E.} \mathrm{F.} \mathrm{L.,} \mathrm{the} \mathrm{probable} \mathrm{errors} \mathrm{are} \mathrm{reduced}$ by one-half. Very few of the lenses plotted were less than 175 $\mathrm{mm}$ E. F. L., while one was as high as $400 \mathrm{~mm}$.

4. Method of Ploting.- The method of plotting the results has been described in the discussion of spherical and sine condition aberrations. The mean values for $V$ and $E$ were so chosen as to show the places of best correction of the lenses in question. Thus, the values of the first two central zones in all cases were disregarded (in taking the mean), as these values are unreliable. In photographic lenses, where no provision is made for the correction of the red rays, the mean of the yellow-green, blue, and violet was taken. On the other hand, in projection and telescope lenses, the violet was disregarded, and the mean of the red, yellow-green, and blue was used. The mean values of $V$ and $E$ were then subtracted from each of the values of $V$ and $E$, respectively, and the resulting differences reduced to $100 \mathrm{~mm}$ focal length. This procedure makes the vertical axis the mean value for both $E$ and $V$. A deviation from the axis means an aberration. The dotted line shows the variation of the E. F. L., and the solid line shows the spherical aberration. The distance between the curves $\Delta E$ and $\Delta V$ is a measure of the coma.

The curves for each wave length are plotted separately, so as not to create confusion, but the vertical axis represents the same line and, for comparison, the figures should be superposed with the axes coinciding, or else the distances from $X Y$ should be compared.

The vertical distances $h$ have been magnified 4 times and the horizontal distances 20 times, in accordance with convention used by Von Rohr and others.

The wave lengths used were:

\begin{tabular}{|c|c|c|}
\hline Limits of band & Mean & Color \\
\hline $423-427 \mu \mu$ (about)... & $\begin{array}{l}\mu \mu \\
425\end{array}$ & Deep violet. \\
\hline $475-479 \mu \mu$ (about)... & 475 & Blue. \\
\hline $547-558 \mu \mu$ (about).... & 550 & Yellow-green. \\
\hline $647-663 \mu \mu$ (about) .............. & 650 & Red. \\
\hline
\end{tabular}


The "limits of band" gives the width of the band of spectrum used for each color.

5. ApPlicability of METHOD. - The method can be successfully used on all optical systems, of relatively large aperture, and short enough focus to permit the plate to be placed sufficiently near the focus to obtain strong light, and still separate the disks a measurable distance. The diameter must be large enough to obtain several zones without crowding the holes in the screen too close together. Such lens systems are photographic lenses, short-focus telescope objectives, étc.

The method has been used on complete instruments, such as telescopes. In a complete telescope, the consideration for a theoretically perfect image requires the rays to be parallel on coming out of the eyepiece. In general, they will not all be

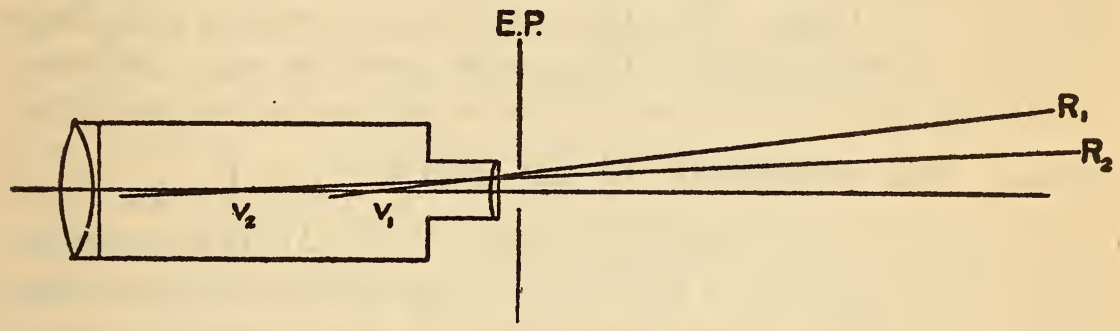

FIG. 10.-Method applied to complete telescope

parallel, but some will intersect the axis at some point behind the exit pupil. If we take two shadowgraphs, one near the exit pupil and one some distance behind it, we can obtain the intersections of the rays with the axis.

Let $R_{1}$ and $R_{2}$ (Fig. Io) be two rays intersecting the axis at distances $V_{1}$ and $V_{2}$ from the rear principal plane of the instrument. Then $U_{1}$ and $U_{2}$ will be such distances that

$$
\begin{aligned}
& \frac{\mathrm{I}}{U_{1}}=\frac{\mathrm{I}}{f}-\frac{\mathrm{I}}{V_{1}} \\
& \frac{\mathrm{I}}{U_{2}}=\frac{\mathrm{I}}{f}-\frac{\mathrm{I}}{V_{2}}
\end{aligned}
$$

where $f$ is the accommodation used by the observer

$$
\frac{\mathrm{I}}{U_{1}}-\frac{\mathrm{I}}{U_{2}}=-\frac{\mathrm{I}}{V_{1}}+\frac{\mathrm{I}}{V_{2}}=-\left(\frac{\mathrm{I}}{V_{1}}-\frac{\mathrm{I}}{V_{2}}\right)
$$

$59466^{\circ}-18-3$ 
The quantity $-\left(\frac{\mathrm{I}}{V_{1}}-\frac{\mathrm{I}}{V_{2}}\right)$ represents the range of accommodation the eye has to assume simultaneously in focussing the rays from the instrument on the retina. Since this is independent of $f$, we see that it does not depend on the observer's eye, nor on the amount of accommodation he requires in using the telescope, nor whether the telescope is focussed on a near object or a distant object, but depends only on the difference in power of the various zones.

6. Illustrative Description of a Set of Curves.-To illustrate the meaning of a set of curves let us take the lens represented by Fig. 25. This is a foreign-made projection lens of the Petzval type for motion-picture projection work. A glance at the curves shows that the lens is best corrected for that region of the spectrum between $475 \mu \mu$ and $550 \mu \mu$, or between the yellowgreen and the blue. Here the dotted curve and the full almost coincide, showing freedom from coma near the axis. At about $13 \mathrm{~mm}$ out from the lens center the curves show appreciable separation. This corresponds to an aperture $I: 3.9\left(\frac{100}{2 \times 13}=\frac{1}{3.9}\right)$. At this aperture the maximum value of $\Delta E-\Delta V$ is $0.09 \mathrm{~mm}$ for wave length $550 \mu \mu$ and $0.05 \mathrm{~mm}$ for wave length $475 \mu \mu$ (disregarding the center zone of the lens); for wave length $425 \mu \mu$, or the violet, the spherical is practiclly zero, but the two curves do not fit so closely, $\Delta E-\Delta V$ becoming equal to $0.15 \mathrm{~mm}$. This is quite small, but it is three times that of wave length $475 \mu \mu$ and twice that of wave length $550 \mu \mu$.

This lens being designed for projection work, the color correction should be between the red and the blue; that is, the red and the blue would focus in the same point and the others not far away, with no attention paid to the extreme violet. The distance of the full line from the axis (the zero line) gives the amount of color aberration for the particular color. The average distances of the four colors from the axis, using the mean of the red, yellowgreen, and the blue as zero, are as follows: Violet, - $0.18 \mathrm{~mm}$; blue, $-0.13 \mathrm{~mm}$; yellow-green, $0.00 \mathrm{~mm}$; and red, +0.22 $\mathrm{mm}$. This shows the red to be far out of achromatisin, as it focusses $0.35 \mathrm{~mm}$ and $0.40 \mathrm{~mm}$ behind the blue and violet, respectively. The progression of the curves in the same direction $(-0.18 \mathrm{~mm}$ to $+0.22 \mathrm{~mm}$ ) shows that no two colors focus at the same point, hence there is no color correction. 
The distances of the dotted curves from the axis shows the variation of the E. F. L. with the color, hence the variation of the size of the image with the color. This is the oblique achromatism. These average distances for each color are as follows: Violet, -0.1 $8 \mathrm{~mm}$; blue, -0.1 $3 \mathrm{~mm}$; yellow-green, +0.06 $\mathrm{mm}$; and red, $+0.25 \mathrm{~mm}$; that is, the red image is 0.4 per cent bigger than the violet, with the sizes of the other images in between. Hence the image projected by this lens shows red fringes on the outside and violet on the inside. This lens could be better used to advantage with a color screen, as each color is well corrected for itself; but the only color for which the variation is small is the blue, and there is no real achromatism as defined earlier in this paper.

The other sets of curves furnish similar information regarding the other lenses, allowing us to draw conclusions as to the best use of the lens as far as the center of the field is concerned.

\section{CLASSIFICATION OF CURVES}

The following curves divide themselves into groups of lenses of several classes. Figs. I I to i 8 represent various types of photographic lenses. The curves here run very smoothly, showing good work in surfacing, as most of these lenses are of a high-grade make. Figs. 14 and 15 are not so regular, but the residuals are so small as to be negligible in any case. Fig. I6 is of an oldtype, rectilinear lens; the rest are modern anastigmats. It will be noticed how the spherical aberration in Fig. I6 does not begin to return to the axis, whereas in the case of the others they either cut the axis again or approach it.

Figs. I9 to 26 are projection lenses of the Petzval type. ${ }^{3}$ The curves are not smooth and regular, showing less care in surfacing than in the photographic objectives. The lenses represented by Figs. I9 to 23 were made in this country, the others being imported. It will be seen that our domestic lenses compare favorably with foreign ones. Figs. 24 and 26 show considerable coma. All the lenses of this group are of rather large aperture, the largest being $f: 2.7$, approximately.

Fig. 27 is a telescope objective (aperture $\mathrm{f}: 3.3$ ) made by Zeiss. The lens is exceedingly well corrected for wave length $550 \mu \mu$, which is the brightest yellow region of the spectrum, with the red and the blue not far away. The violet has been entirely

\footnotetext{
${ }^{8}$ First brought out by $\mathrm{J}$. Petzval in 1840 . It is described in all books on optical instruments.
} 
neglected. This is also true of the color correction of the projection lenses, as they all have to be used for visual work.

\section{SUMMARY}

The errors which affect the definition of a lens are discussed and methods of representing graphically the central errors described. The condition for freedom from coma near the axis is arrived at. The relative importance of the errors in the different types of lenses is discussed. Hartmann's method is extended, permitting one set of measurements to give all the important central errors-spherical aberration, zonal variation of the equivalent focal length, axial and oblique achromatism. The apparatus and the procedure are described, and the accuracy of the adjustments and measurements discussed. The method is applicable to all systems of relatively short focus and large aperture, such as photographic lenses, projection lenses, and telescope objectives, and also to complete optical systems. The results of the method as applied to a complete telescope are discussed, and are shown to be independent of the accommodation of the observer. Seventeen sets of curves for as many different lenses are given, and an illustrative discussion of one set of curves, together with a general description of the types of lenses represented by each group of curves.

Washington, September i9, igi6. 


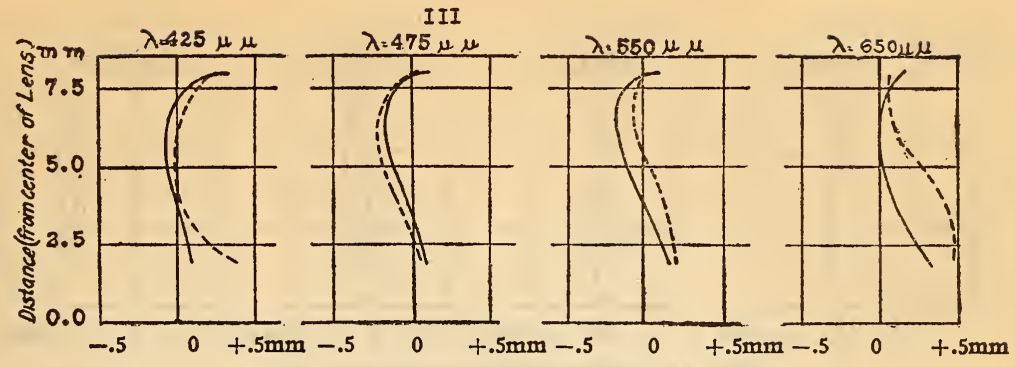

FIG. II.-Photographic anastigmat lens
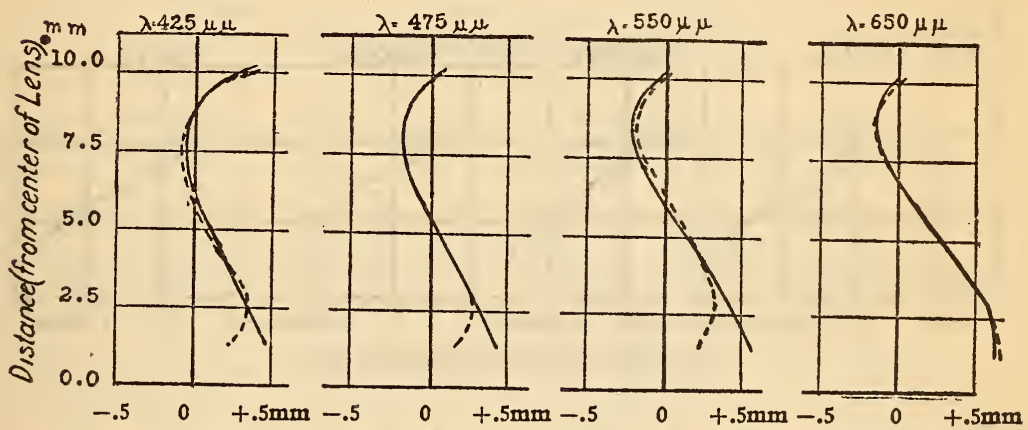

FIG. 12.-Photographic anastigmat lens
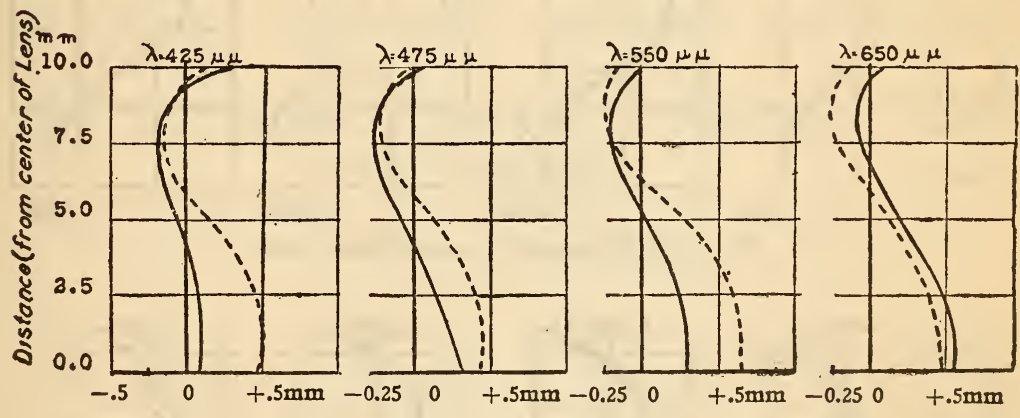

FIG. I3.-Photographic anastigmat lens
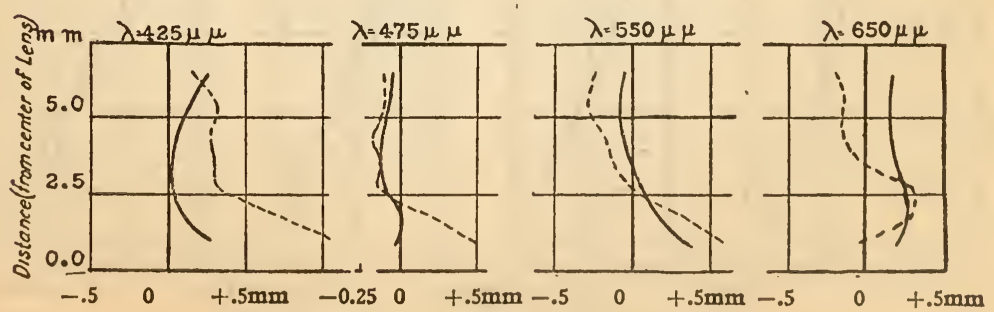

FIG. I4.-Photographic anastigmat lens

Spherical aberration

Sine condition 


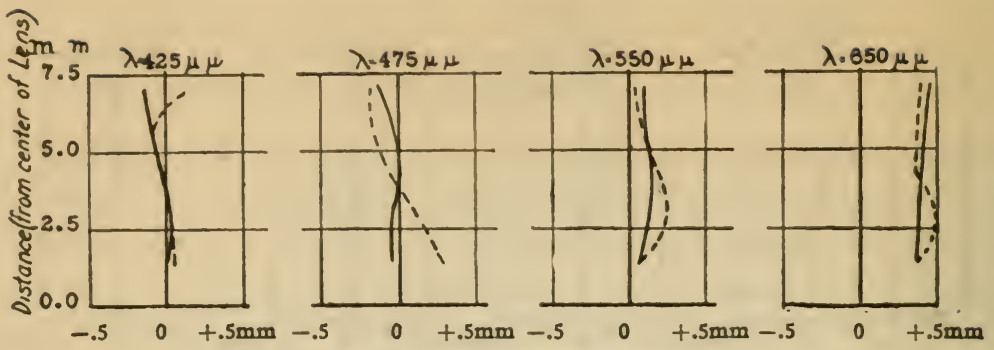

FIG. I5.-Photographic anastigmat lens

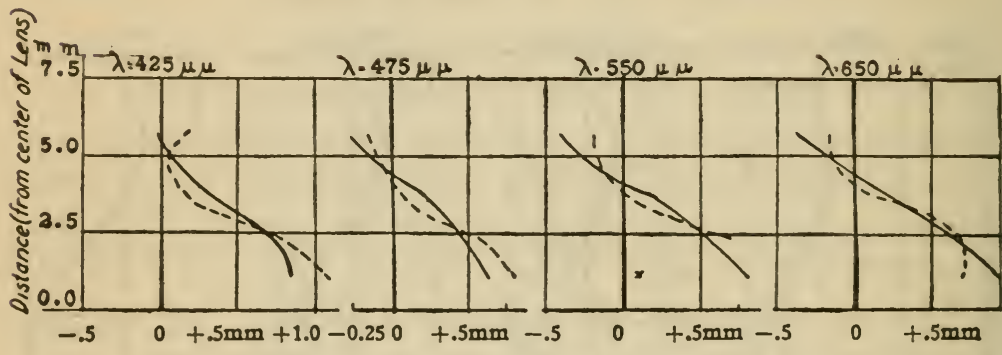

Fig. I6.-Old type Rectihnear lens
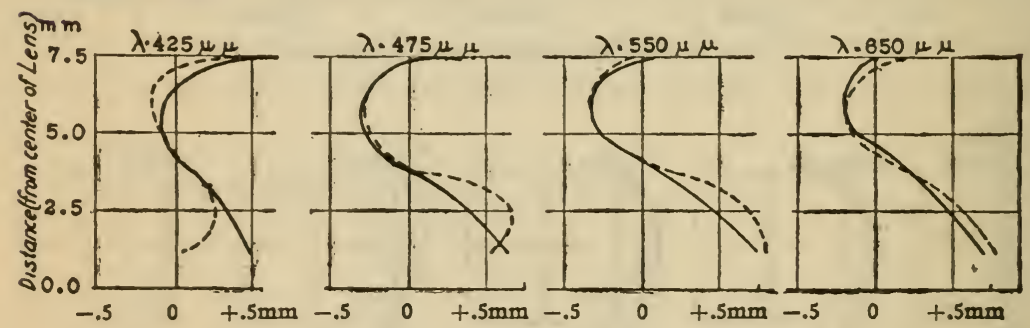

FIG. 17.-Photographic anastigmat lens
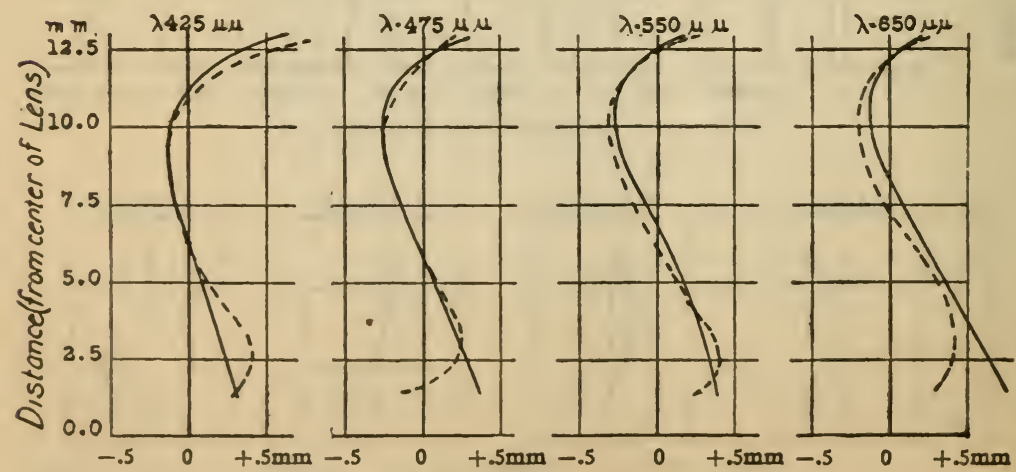

FIG. 18.-Photographic anastigmat lens

Spherical aberration

Sine condition 

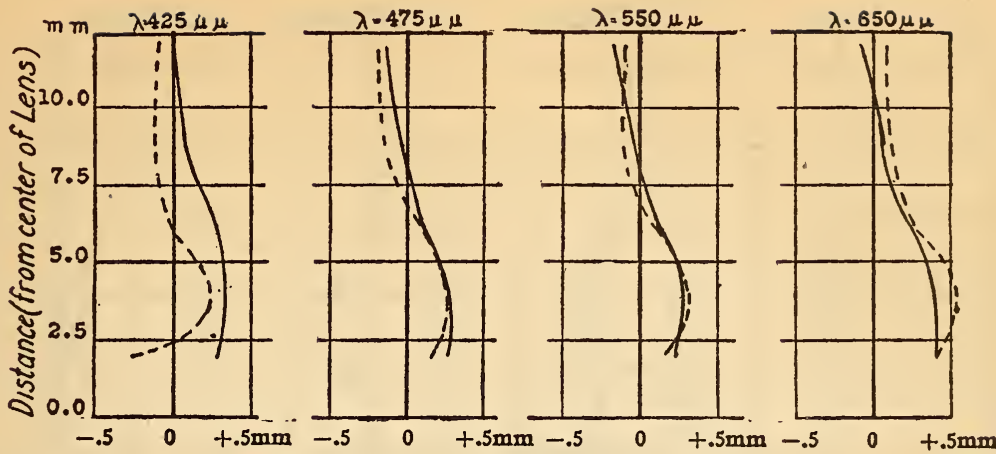

FIG. 19.-Projection lens
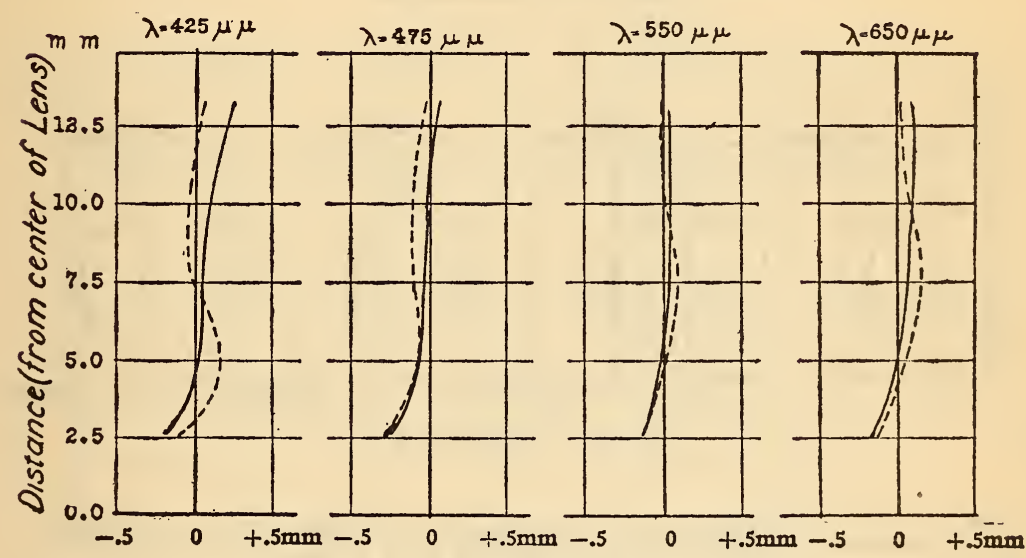

Fig. 20.-Projection lens
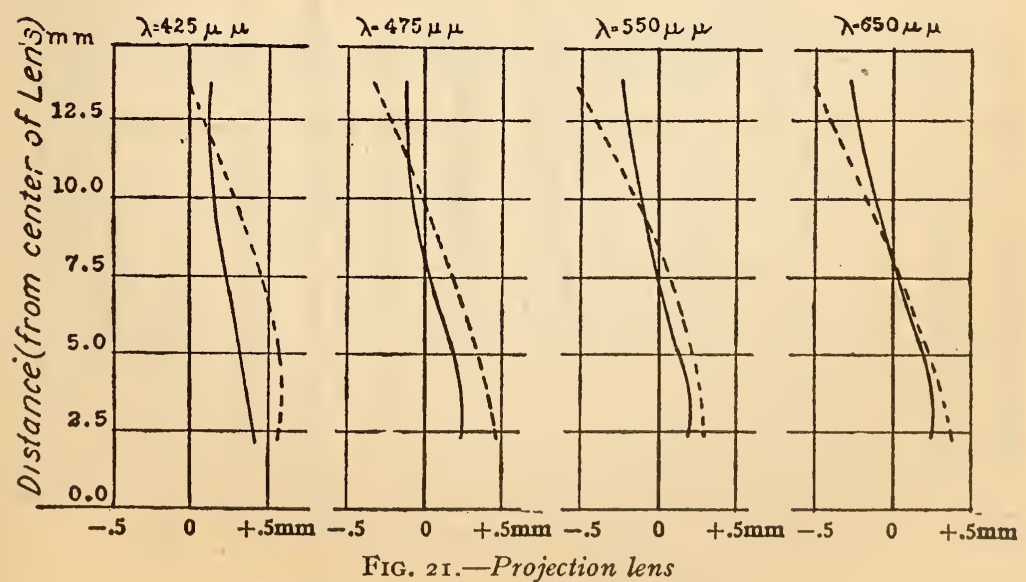

Spherical aberration

Sine condition 

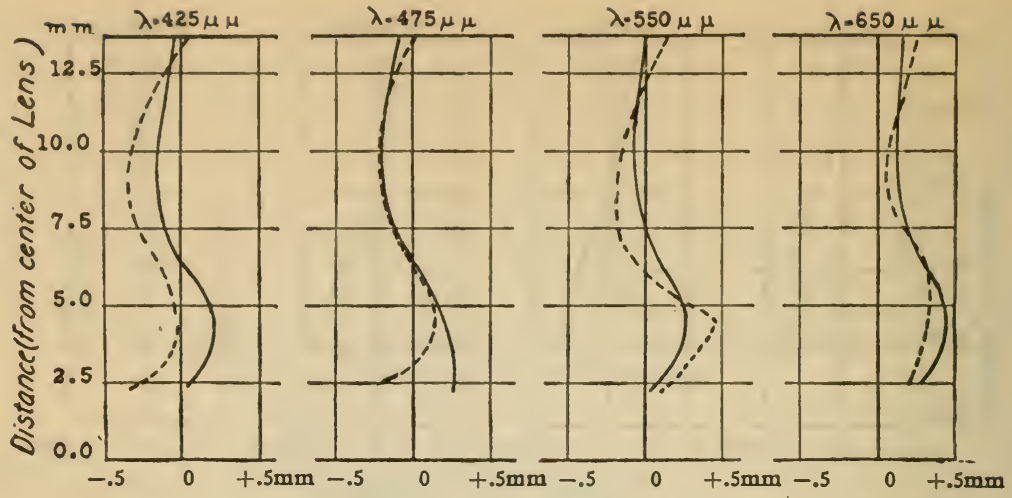

FIG. 22.-Projection lens
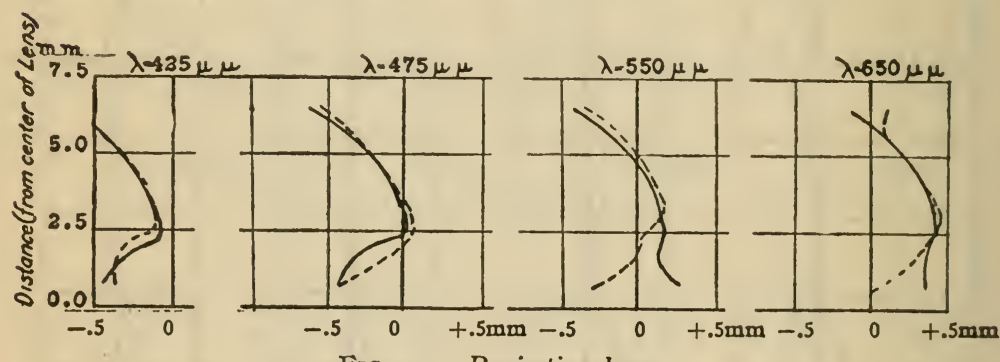

FIG. 23.-Projection lens

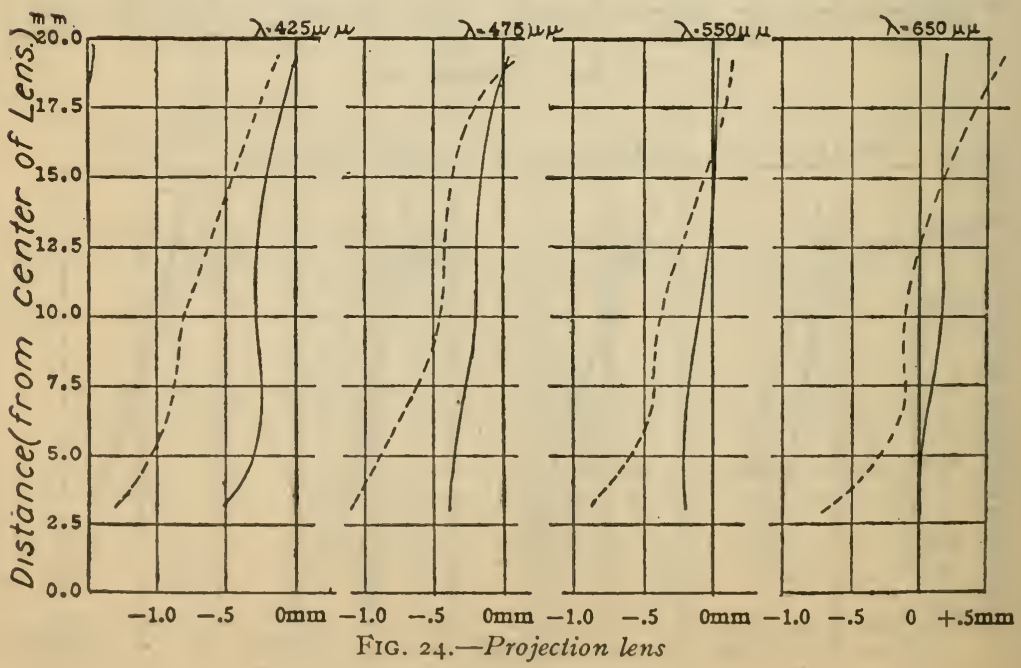

Spherical aberration

Sine condition 

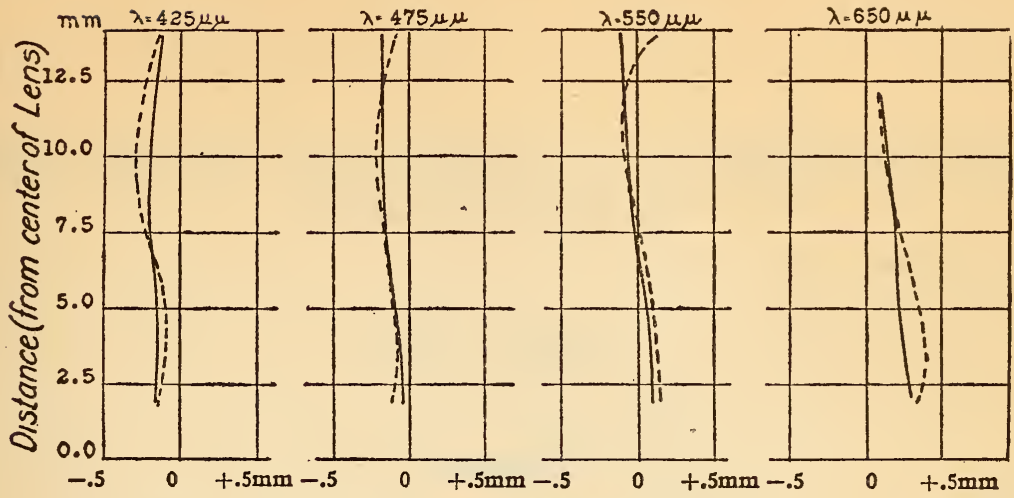

FIG. 25.-Projection lens
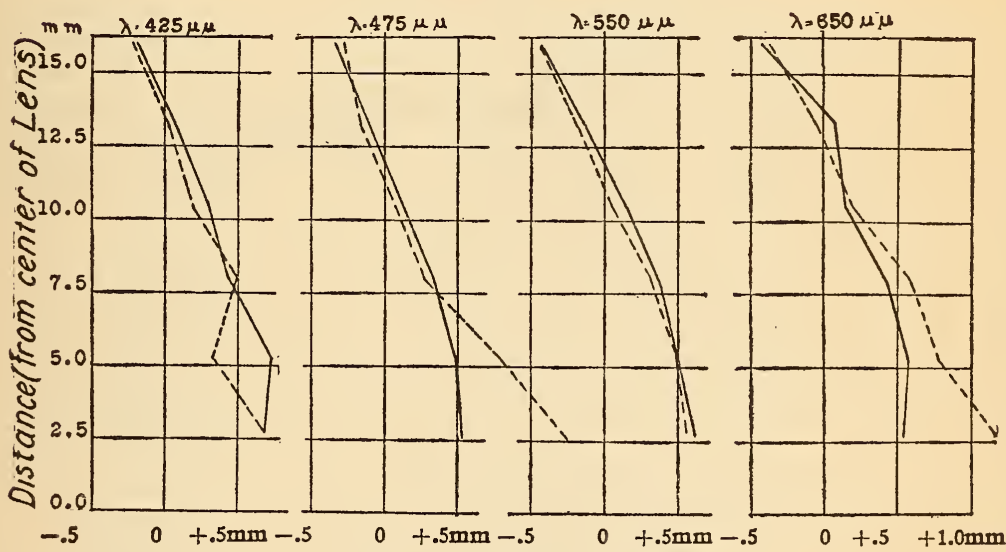

Fig. 26.-Projection lens

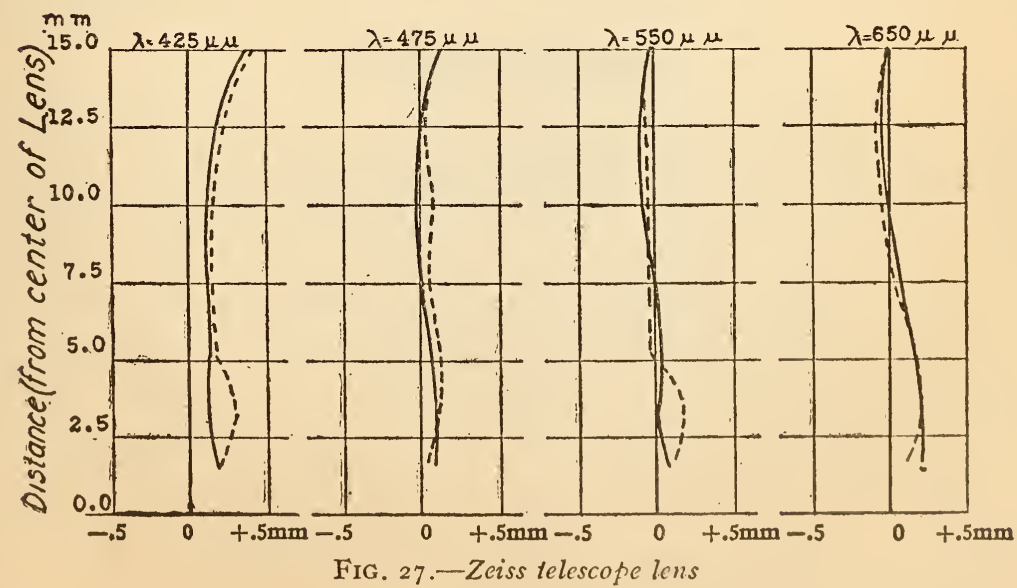

Spherical aberration

Sine condition 